\title{
Targeting the PD-L1/DNMT1 axis in acquired resistance to sorafenib in human hepatocellular carcinoma
}

\author{
JIANHUA LIU, YAHUI LIU, LINGYU MENG, KAI LIU and BAI JI \\ Department of Hepatobiliary and Pancreatic Surgery, The First Hospital of Jilin University, \\ Changchun, Jilin 130021, P.R. China
}

Received January 18, 2017; Accepted June 8, 2017

DOI: $10.3892 /$ or.2017.5722

\begin{abstract}
Molecule-targeted therapy, such as sorafenib, is one of the effectively therapeutic options for advanced hepatocellular carcinoma (HCC). However, acquired resistance to sorafenib has been found in some HCC patients, resulting in poor prognosis. It is reported that PD-L1 and DNA methyltransferases (DNMTs) contribute to drug resistance. In this study, by inducing sorafenib-resistant HCC cell lines, we investigated their molecular and functional characteristics. Our data indicated that highly upregulated DNMT1 was positively correlated with PD-L1 overexpression in sorafenib-resistant HCC cells. We demonstrate that PD-L1 regulate DNMT1 through STAT3 signaling pathway. Knockdown of PD-L1 induced DNMT1dependent DNA hypomethylation and restored the expression of methylation-silenced CDH1. Moreover, inactivation of NFאB blocked PD-L1/STAT3/DNMT1 pathway in sorafenibresistant HCC cells. Functionally, genetic or pharmacological disruption of PD-L1 or/and DNMT1 sensitize HCC resistance to sorafenib. Importantly, dual inactivation of PD-L1 and DNMT1 by their inhibitor synergistically disrupts the colony formation of sorafenib-resistant HCC cells. These results demonstrate that targeting NFאB/PDL1/STAT3/DNMT1 axis is a new therapeutic strategy for preventing or overcoming the acquired resistance to sorafenib in $\mathrm{HCC}$ patients.
\end{abstract}

\section{Introduction}

Hepatocellular carcinoma (HCC) is one of the most frequently diagnosed cancer across the globe, and the most common primary liver tumor with increasing incidence worldwide. Targeted therapy is one of the effectively therapeutic options for advanced HCC during the past few years (1). Sorafenib is a multitargeted tyrosine kinase inhibitor for the treatment of

Correspondence to: Dr Bai Ji, Department of Hepatobiliary and Pancreatic Surgery, The First Hospital of Jilin University, 71 Xinmin Street, Changchun, Jilin 130021, P.R. China

E-mail: jirulin@sina.com

Key words: hepatocellular carcinoma, sorafenib resistance, PD-L1, DNMT1, DNA methylation
HCC that blocks the Ras, VEGFR, PDGFR, FLT3 and KIT kinases, which increases the rate of apoptosis and inhibits cell proliferation, migration and tumor angiogenesis (2). However, acquired resistance to sorafenib has been found in HCC patients, which results in poor prognosis. The limited survival benefit from these clinical trials suggests the existence of primary and acquired sorafenib resistance mechanisms in HCC cells. Recently, some studies report novel molecular mechanism of sorafenib resistance in HCC cells. A low-molecular weight and cysteine-rich proteins, metallothionein (MT)-1G is reported as a critical regulator and promising therapeutic target of sorafenib resistance in human HCC cells (3). Using in vivo RNAi screening, Rudalska et al suggest that Mapk14 blockade is a promising approach to overcoming therapy resistance of human HCC (4). Tumor-associated neutrophils (TANs) mediated the intratumoral infiltration of macrophages and Treg cells by secreting CCL 2 and CCL17, which stimulated neovascularization, enhanced HCC growth and metastasis, and contributed to sorafenib resistance, suggesting that TAN depletion could enhance the efficacy of sorafenib as an anti-HCC therapeutic (5). However, the resistance mechanism remains poorly understood.

Tumor cells often overexpress immune checkpoint proteins to allow them to evade the host immune system by inhibiting T-cell attack. One of these immune checkpoint proteins is programmed death-ligand-1 (PD-L1), which binds to programmed death-1 (PD-1) expressed on T-cells, B-cells, dendritic cells and natural killer T-cells to suppress anticancer immunity (6). Therefore, anti-PD-L1 and anti-PD-1 antibodies have been used for the treatment of cancer, showing promising outcomes $(7,8)$. Moreover, PD-L1 also overexpressed drug-resistant cancer cells, such as enzalutamide-resistant prostate cancer (9), cisplatin-resistant small cell lung cancer cells (10). Despite the importance of PD-L1 in tumor immunity and drug resistance, the regulation of PD-L1 expression remains poorly understood. Zhu et al found PDL1 is a direct target of BRD4-mediated gene transcription. BET inhibitors suppress PD-L1 expression in both immune cells and ovarian cancer cells (11). Hypoxia upregulates PD-L1 on mouse and human tumor cell lines and on macrophages and DCs from naive C57BL/6 mice (12). Transcriptional factor MYC directly regulates CD47 and PD-L1 at the transcriptional level by binding to their promoters in human melanoma (13). Lo et al showed that inflammation increases PD-L1 expression in 
tumors through $\mathrm{TNF}-\alpha$-mediated activation of $\mathrm{NF} \kappa \mathrm{B}$, leading to transactivation of CSN5 (14).

Epigenetic changes such as DNA methylation act to regulate gene expression in normal mammalian development. However, promoter hypermethylation also plays a major role in cancer through transcriptional silencing of critical growth regulators such as tumor suppressor genes (15). DNA methylation is controlled at several different levels in normal and tumor cells. The addition of methyl groups is carried out by a family of enzymes, DNA methyltransferases (DNMTs). DNMTs are enzymes that catalyze the addition of methyl groups to cytosine residues in DNA. DNMTs found in mammalian cells include DNMT1, DNMT3a, and DNMT3b $(16,17)$.

In this study, we modeled sorafenib resistance in HCC cell lines, and explored the molecular and functional characteristics of resistant cells. We demonstrate NFאB/PDL1/STAT3/ DNMT1 axis as a mechanism by which HCC cells develop sorafenib-resistance phenotypes and established its potential as a new therapeutic target for preventing or overcoming the acquired resistance to sorafenib.

\section{Materials and methods}

Plasmids, cell lines and chemicals. The shRNA and control vectors for PD-L1, DNMT1, STAT3 and NFאB were obtained from BMGC RNAi (University of Minnesota). Cell lines were obtained from American Type Culture Collection (Manassas, VA, USA). HepG2 and Huh7 cell lines were grown in DMEM with $10 \%$ fetal bovine serum (FBS) (Life Technologies, Grand Island, $\mathrm{NY}, \mathrm{USA}$ ) at $37^{\circ} \mathrm{C}$ under $5 \% \mathrm{CO}_{2}$. For the drug treatment, cells were treated with the following reagents used at concentrations, times and schedules indicated in Results. Sorafenib, decitabine (5-aza-2'-deoxycytidine or Dacogen) and Bay 11-7082 were purchased from Sigma-Aldrich (St. Louis, MO, USA).

Generation of sorafenib-resistant cells. HepG2 and Huh7 sorafenib-resistant cells (HepG2 $2^{\mathrm{SR}}$ and Huh7 ${ }^{\mathrm{SR}}$ ) were cultured continuously with a step-wise increase of sorafenib concentrations for 8 weeks $(0-20 \mu \mathrm{M})$. HepG2 and Huh7 parental cells (HepG2 $2^{\mathrm{C}}$ and $H u h 7^{\mathrm{C}}$ ) were cultured in parallel without sorafenib and served as control.

Transfections. Approximately $1 \times 10^{6}$ cells were seeded into 6-well plates overnight before transfection. The shRNA or plasmids were introduced into cells using Lipofectamine ${ }^{\mathrm{TM}}$ RNAiMAX or Lipofectamine $^{\mathrm{TM}} 2000$ reagent (Life Technologies), respectively, according to the manufacturer's instructions.

Chromatin immunoprecipitation (ChIP). ChIP assays were performed as described previously using EZ-ChIP assay kit (Millipore, Billerica, MA, USA). Briefly, $\sim 2 \times 10^{6}$ transfected cells were cross-linked with $1 \%$ formaldehyde (SigmaAldrich), washed and resuspended in 1\% SDS lysis buffer for sonication, in order to yield DNA fragments with an average size of 300-500 bp. The lysates were immunoprecipitated by $5 \mu \mathrm{g}$ of antibody. Aliquots (1\%) were reserved for the negative control (input DNA). ChIP DNA was quantified by qPCR with Power SYBR ${ }^{\circledR}$ Green PCR Master Mix. Fold change in binding was compared using the corresponding input DNA. The primers specific for DNMT1 or PDL1 gene promoter were: hDNMT1-ChIP1 forward, AATAGATGGAGGTTGGAT; reverse, AGGCATTCATTCATTCAT. hDNMT1-ChIP2 forward, CTATACACTGTGAGATTCTTG; reverse CTGGC TATACGACCTTAG. The anti-STAT3, anti-phospho-STAT3

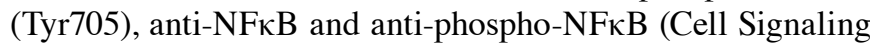
Technology) were used.

Clonogenic assays. Methylcellulose colony formation assays were performed in MethoCult ${ }^{\circledR}$ medium (Stem Cell Technologies, Canada) according to the manufacturer's instructions. Briefly, at $6 \mathrm{~h}$ after transfection or exposure to drugs, 500 cells were harvested and diluted in $0.3 \mathrm{ml}$ of IMDM $+2 \%$ FBS (Stem Cell Technologies), then mixed diluted cells in MethoCult ${ }^{\circledR}$ medium. Subsequently $1.1 \mathrm{ml}$ of the MethoCult mixture was dispensed into a $35-\mathrm{mm}$ dish. Colonies were scored in 7-10 days.

Western blotting. After the various treatments, the whole cellular lysates were prepared by harvesting the cells in $1 \mathrm{X}$ cell lysis buffer [20 mM HEPES (pH 7.6), $150 \mathrm{mM}$ $\mathrm{NaCl}$ and $0.1 \% \mathrm{NP} 40]$ supplemented with $1 \mathrm{X}$ phosphatase inhibitor Cocktail 2 and 3 (Sigma-Aldrich), $1 \mathrm{mM}$ PMSF (Sigma-Aldrich) and $1 \mathrm{X}$ protease inhibitors (protease inhibitor cocktail set III, Calbiochem-Novabiochem, San Diego, CA, USA). Protein was resolved by sodium dodecyl sulfate (SDS)-polyacrylamide gel electrophoresis and transferred onto PVDF membranes (Amersham, Piscataway, NJ, USA). The antibodies used were $\beta$-actin (Santa Cruz Biotechnology); DNMT1 (New England Biolabs, Ipswich, MA, USA), the anti-STAT3, anti-phospho-STAT3 (Tyr705),

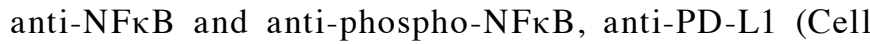
Signaling Technology).

RNA isolation, cDNA preparation and quantitative PCR. RNA was isolated using miRNAeasy kit (Qiagen) according to the manufacturer's instructions. Reverse transcription for obtaining cDNA was performed according to the manufacturer's instructions (Invitrogen). The expression of DNMT1, $P D-L 1, C D H 1$ and GAPDH gene was evaluated by SYBR Green Quantitative PCR. Expression of the target genes was measured using the $\Delta \mathrm{CT}$ approach. The primers were: DNMT1 forward, 5'-CCAGATGAGGACAATGAG-3'; reverse, 5'-AGCAAGACAACCATAATCA-3'. PD-L1 forward, 5'-TCC ACTCAATGCCTCAAT-3'; reverse, 5'-GAAGACCTCACA GACTCAA-3'. GAPDH forward, 5'-ACAGGATTGACAGA TTGA-3'; reverse, 5'-TATCGGAATTAACCAGACA-3'. CDH1 forward, 5'-AGAACGCATTGCCACATACAC-3'; reverse, GAGGATGGTGTAAGCGATGG-3'.

Bisulfite sequencing. Total DNA sample $(2 \mu \mathrm{g})$ was converted and purified using EpiTect Bisulfite kit (Qiagen) according to the manufacturer's instructions. The -251 to +139 region within the CDH1 CpG was amplified from bisulfite-treated DNA sample by PCR using the following primers: forward, 5'-TTTTTTTTGATTTTAGGTTTTAGTGAG-3'; reverse, 5'-ACTCCAAAAACCCATAACTAACC-3'. The PCR products were subcloned using the TA Cloning ${ }^{\circledR}$ kit (Invitrogen), and sequenced by Genewiz Company. 


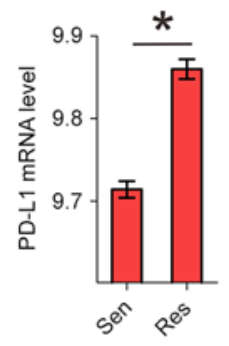

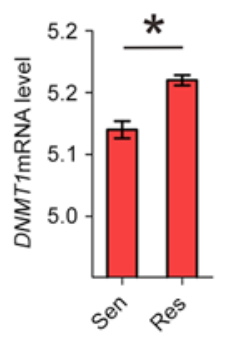

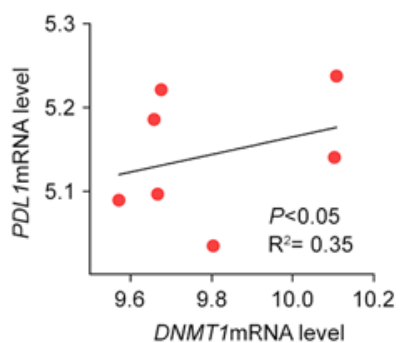

C

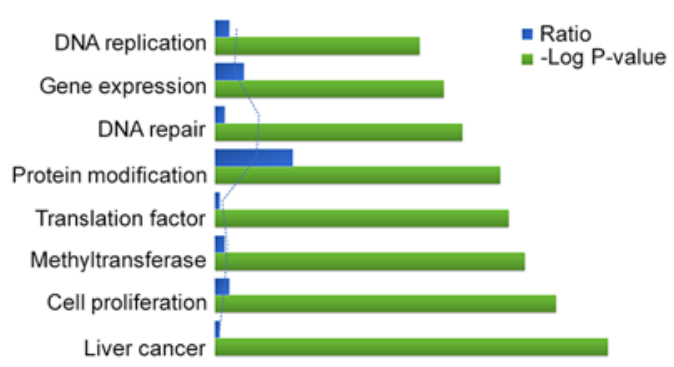

D

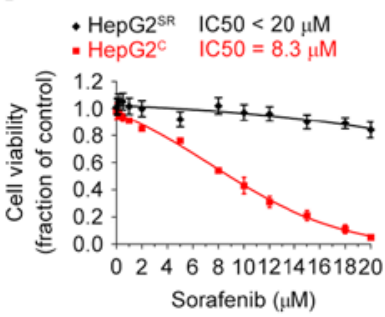

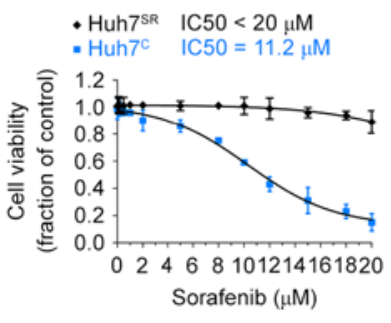

$\mathrm{E}$

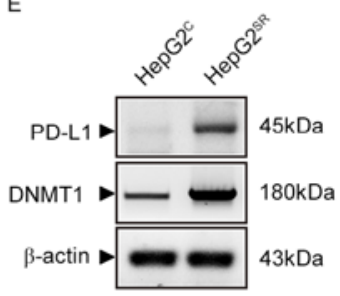

$\mathrm{F}$
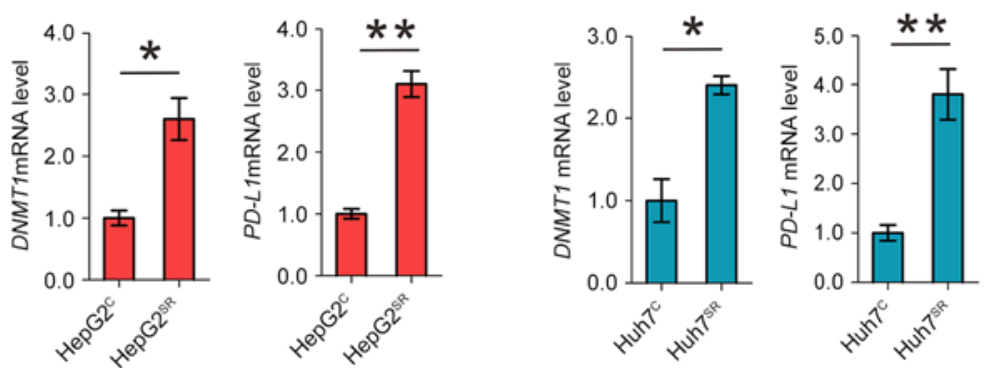

Figure 1. PD-L1 and DNMT1 are frequently overexpressed and positively correlated in HCC resistance to sorafenib. (A) GEO data were analyzed for PD-L1 and DNMT1 expression in sorafenib-sensitive mice versus sorafenib-resistant mice. (B) The analysis of GEO dataset GSE73571 showing the correlation between PD-L1 and DNMT1 expression in sorafenib-resistant mice, GSE73571. Correlation between PD-L1 and DNMT1 was assessed by Pearson correlation. $\mathrm{P}<0.05$ was considered statistically significant. (C) Enrichment scores for Gene Ontology (GO) categories in overlapped genes. The -log (P-value) axis indicates the statistical significance of the functions to the dataset. (D) Sorafenib-resistant cells were treated with sorafenib for $72 \mathrm{~h}$. The cell proliferation was assessed by CCK-8 assays. (E) Western blotting for PD-L1 and DNMT1 expression in HepG2 ${ }^{\mathrm{C}}$ and $\mathrm{HepG}^{\mathrm{SR}}$ cells. (F) qPCR measuring the expression levels of indicated genes in $\mathrm{HepG}_{2}{ }^{\mathrm{C}}$ and $\mathrm{Huh}^{\mathrm{C}}$ vs HepG2 ${ }^{\mathrm{SR}}$ and Huh7 ${ }^{\mathrm{SR}}$ cells. Data are mean $\pm \mathrm{SD},{ }^{*} \mathrm{P}<0.05,{ }^{* *} \mathrm{P}<0.01$. The data represent three independent experiments.

Statistical analysis. The qPCR and colony assay were analyzed using the Student's t-test. Correlation data were performed with Pearson correlation coefficients. The statistical analysis were carried out using GraphPad Prism 5.0. Differences were considered statistically significant at $\mathrm{P}<0.05$. All $\mathrm{P}$-values were two-tailed.

\section{Results}

Highly upregulated DNMT1 is positively correlated with $P D$-L1 overexpression in sorafenib-resistant HCC cells. To determine whether PD-L1 and DNMT1 expression is upregulated in sorafenib-resistant HCC cells, we analyzed the GEO data discovered a significant PD-L1 and DNMT1 upregulation in sorafenib-resistant HCC mice (Fig. 1A). In addition, as shown in Fig. 1B, we found that in HCC mice, higher levels of DNMT1 are accompanied by PD-L1 overexpression, while lower expression of DNMT1 is observed in mice carrying lower PDL1 mRNA level, indicating of a positive correlation between these variables $(\mathrm{P}<0.05)$. To better understand which biological functions are affected by long-term sorafenib treatment, we conducted functional annotations using DAVID bioinformatics resources 6.7. Enrichment scores for Gene Ontology (GO) categories in overlapped genes (Fig. 1C). To further understand the sorafenib resistance mechanism, we established two HCC sorafenib-resistant cell lines, HepG2 ${ }^{\mathrm{SR}}$ and Huh7 ${ }^{\mathrm{SR}}$, by the stepwise increase of drug dosages and continuous culture in drug-containing medium for 2 months. The final concentrations were $20 \mu \mathrm{M}$ of sorafenib, which exerted sufficient inhibitory action and were in the range of clinically achievable levels (18). HepG $2^{\mathrm{SR}}$ and $\mathrm{Huh} 7^{\mathrm{SR}}$ exhibited significantly higher $\mathrm{IC}_{50}$ value than their parental control HepG2 $2^{\mathrm{C}}$ and Huh7 ${ }^{\mathrm{C}}$ (Fig. 1D). Notably, comparing to the parental cells, the protein expression and RNA level of DNMT1 and PD-L1 were all increased in HepG2 $2^{\mathrm{SR}}$ and Huh7 $7^{\mathrm{SR}}$ (Fig. 1E and F). These results support the highly upregulated DNMT1 is positively correlated with PD-L1 overexpression in sorafenib-resistant HCC cells.

PD-L1 regulates DNMT1 through STAT3 signaling pathway. Given the positive correlations of DNMT1 and PD-L1 expression in sorafenib-resistant HCC, we proposed the existence 

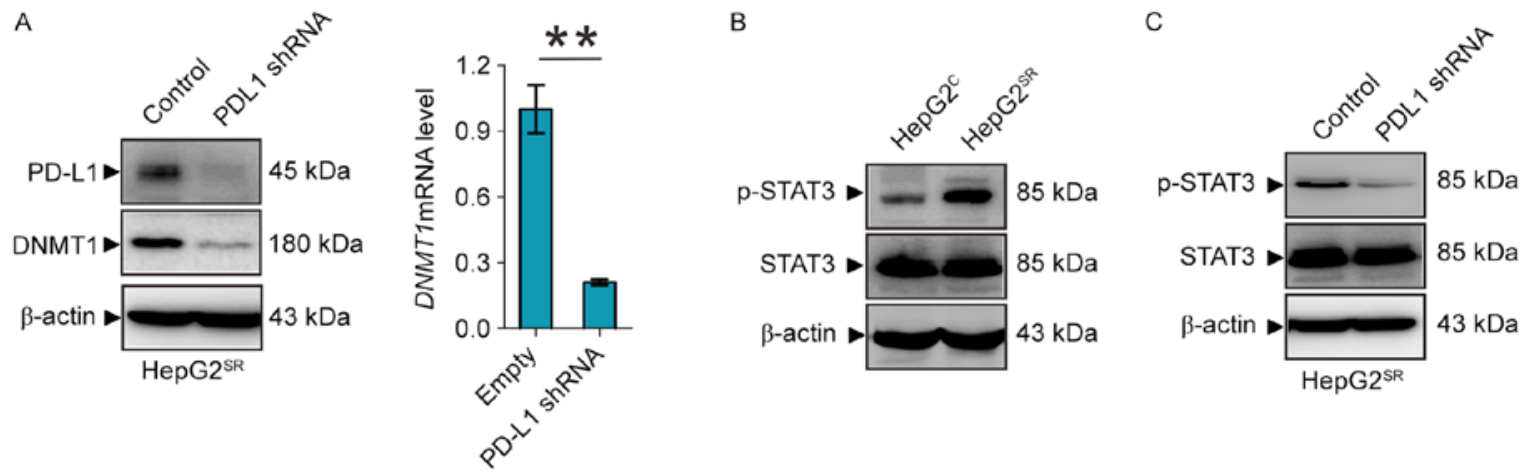

D
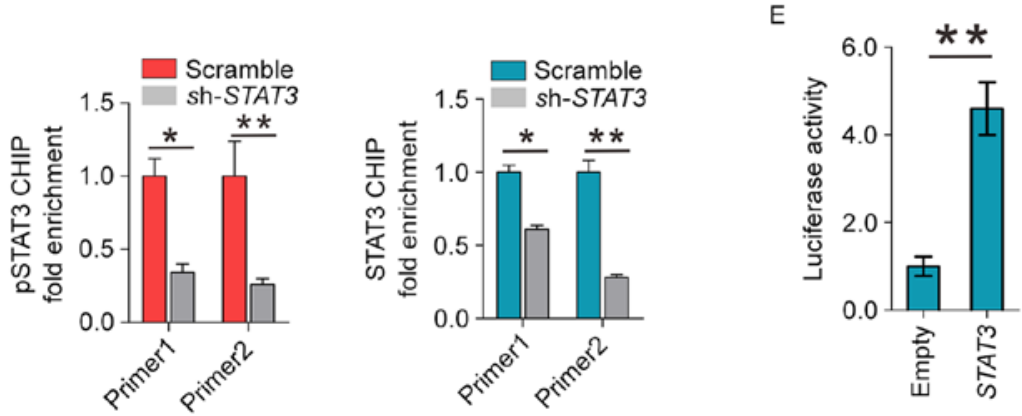

Figure 2. PD-L1 regulates DNMT1 through STAT3 signaling. (A) Western blotting (left) and qPCR (right) for DNMT1 and PD-L1 in HepG2 ${ }^{\mathrm{SR}}$ transfected with PD-L1 empty vector or shRNA. (B and C) Western blotting for the protein expression of p-STAT3 and total STAT3 in HepG2 $2^{\mathrm{SR}}$ and $\mathrm{HepG}_{2}^{\mathrm{C}}(\mathrm{B})$ or HepG2 ${ }^{\mathrm{SR}}$ transfected with PDL1 empty vector or shRNA (C). (D) HepG2 ${ }^{\text {SR }}$ cells were transfected with STAT3 siRNA or its control vector for $48 \mathrm{~h}$ and subjected to ChIP. The change of STAT3 binding on DNMT1 promoter was assessed by qPCR. (E) 293T cells were transfected with pGL3-DNMT1 alone or plus STAT3 vectors for $48 \mathrm{~h}$, followed by the measurement of luciferase activity. Data are mean $\pm \mathrm{SD},{ }^{,} \mathrm{P}<0.05,{ }^{* *} \mathrm{P}<0.01$. The data represent three independent experiments.

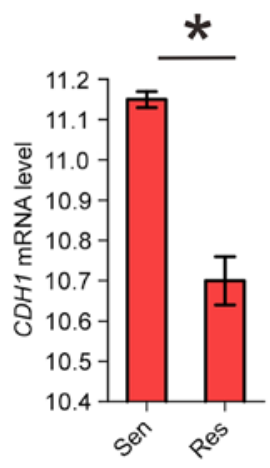

C

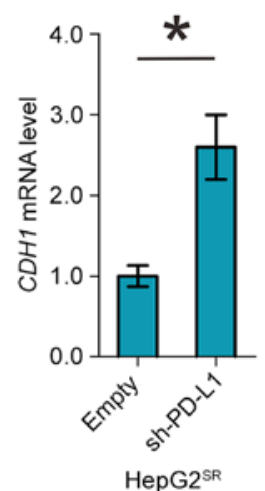

B
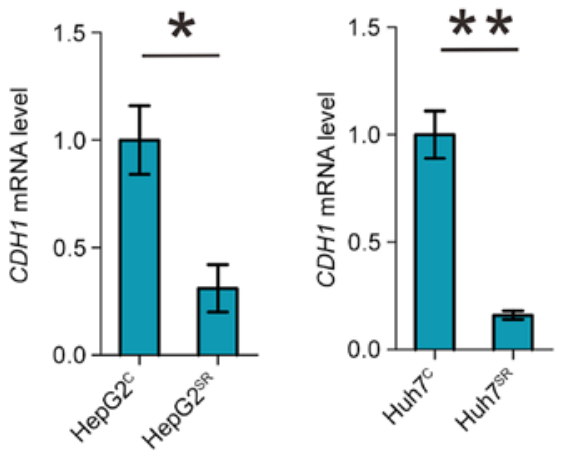

D

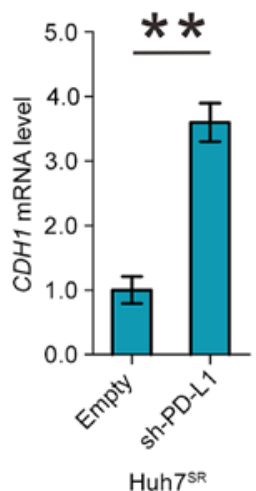

$\mathrm{CDH} 1 \mathrm{CpG}$

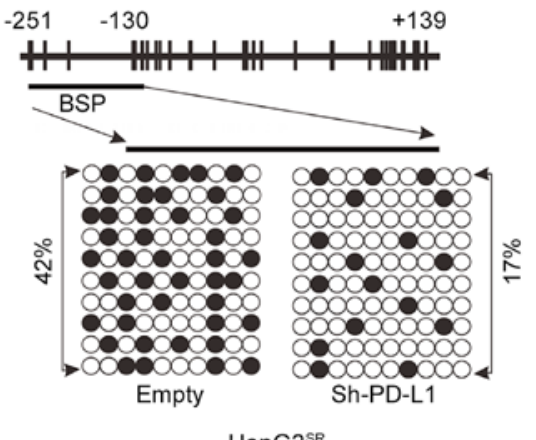

HepG2 $2^{\text {SR }}$

Figure 3. PD-L1 induces DNMT1-dependent DNA hypomethylation and restores the expression of methylation-silenced CDH1. (A) GEO data were analyzed for $\mathrm{CDH} 1$ expression in sorafenib-sensitive mice versus sorafenib-resistant mice. (B) qPCR measuring the expression levels of CDH1 genes in HepG2 ${ }^{\mathrm{C}}$ and $\mathrm{Huh}^{\mathrm{C}}$ vs HepG2 ${ }^{\mathrm{SR}}$ and $\mathrm{Huh} 7^{\mathrm{SR}}$ cells. (C) $\mathrm{qPCR}$ for CDH1 expression in $\mathrm{HepG}_{2}^{\mathrm{SR}}$ and Huh7 ${ }^{\mathrm{SR}}$ cells transfected with empty or PD-L1 shRNA. (D) Bisulfite analysis for the change of DNA methylation in CDH1 promoter (transcription start site -251 to +139 ) in HepG2 ${ }^{\text {SR }}$ cells transfected with empty or PD-L1 shRNA. Data are mean $\pm \mathrm{SD},{ }^{*} \mathrm{P}<0.05,{ }^{* * *} \mathrm{P}<0.01$. The data represent three independent experiments. 
A

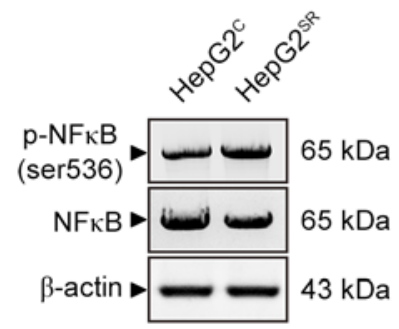

B

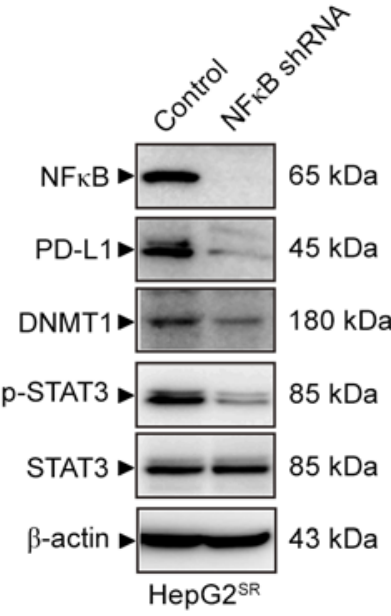

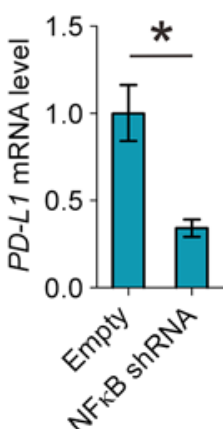

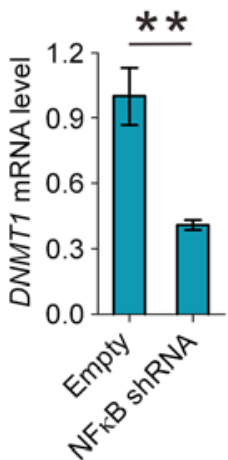

D

C

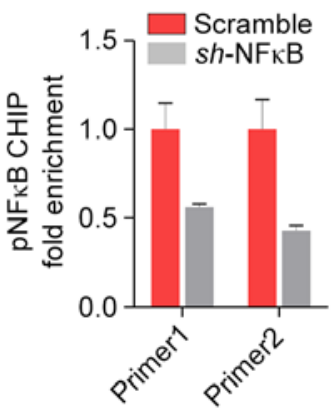

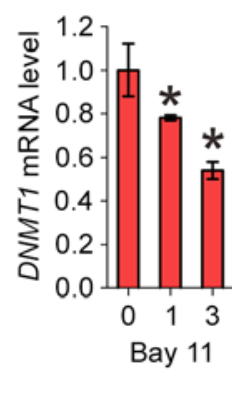

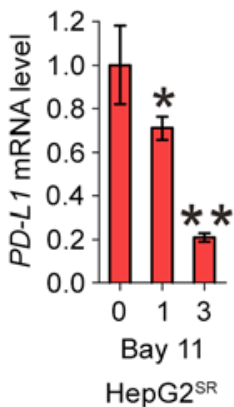

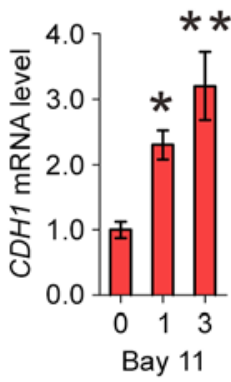

Figure 4. Inactivation of NFKB blocks PD-L1/Stat3/DNMT1 pathway in sorafenib-resistant HCC cells. (A) Western blotting for the protein expression of

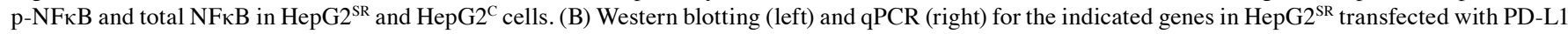
empty vector or shRNA. (C) HepG2 $2^{\text {SR }}$ cells were transfected with NFkB shRNA or its control vector for $48 \mathrm{~h}$ and subjected to ChIP. The change of NFkB binding on PD-L1 promoter was assessed by qPCR. (D) qPCR for DNMT1, PDL1 and CDH1 levels in HepG2 ${ }^{\text {SR }}$ cells treated with Bay 11 for 48 h. Data are mean $\pm \mathrm{SD},{ }^{*} \mathrm{P}<0.05,{ }^{* *} \mathrm{P}<0.01$. The data represent three independent experiments.

of a regulatory loop between DNMT1 signaling and PD-L1 machinery, in which $\mathrm{PD}-\mathrm{L} 1$ regulates the expression of DNMT1. As shown in Fig. 2A, PD-L1 depletion by shRNA in HepG $2^{\text {SR }}$ and Huh7 ${ }^{\text {SR }}$, as expected, decrease PD-L1 and DNMT1 protein and mRNA levels. To elucidate how PD-L1 regulates the DNMT1 gene, we focused on the STAT3/ DNMT1 network, since STAT3 has been shown to regulate DNMT1 transcription in cancer and play a key role in drugresistant (19). First, we found that phosphor-STAT3 was overexpressed in HepG2 ${ }^{\text {SR }}$ compared with HepG2 ${ }^{\mathrm{C}}$ (Fig. 2B). Second, PD-L1 knockdown dephosphorylates STAT3 at Tyr705 in HepG $2^{\text {SR }}$ and Huh7 ${ }^{\mathrm{SR}}$ (Fig. 2C). In addition, ChIP showed that STAT3 knockdown diminished the binding of total and phospho-STAT3 in DNMT1 promoter (Fig. 2D). Reporter assays revealed that STAT3 inactivation by shRNA disrupted the luciferase activities driven by DNMT1 promoter region containing STAT3 binding elements (Fig. 2E). These results confirmed STAT3 transcript regulates the DNMT1 gene, and suggest that PD-L1 specifically regulate DNMT1 through STAT3 signaling in sorafenib resistance to HCC.

PD-L1 induces DNMT1-dependent DNA hypomethylation and restores the expression of methylation-silenced $\mathrm{CDH}$. Cadherin 1 (CDH1) is a cell-cell adhesion molecule and functions as a metastasis suppressor in HCC. It is epigenetically silenced and its downregulation associates with poor prognosis in HCC (20,21). As shown in Fig. 3A and B we found significant CDH1 downregulation in sorafenib-resistant HCC mice and cell model. Important, knockdown of PDL1 can increase CDH1 mRNA level in HepG2 ${ }^{\mathrm{SR}}$ and Huh7 ${ }^{\mathrm{SR}}$ cells (Fig. 3C). As PD-L1 regulates DNMT1, we proposed that PD-L1 downregulation might mediate $\mathrm{CDH} 1$ restoration via promoter DNA hypermethylation. We analyzed $\mathrm{CDH} 1$ promoter methylation status using bisulfite sequencing in PD-L1 shRNA-transfected HepG2 ${ }^{\text {SR }}$ and Huh7 ${ }^{\text {SR }}$ cells, and found a $>25 \%$ change ( $42 \%$ in empty versus $17 \%$ in PDL1 shRNA) from hyper- to unmethylated in CDH1 promoter (Fig. 3D). Thus, PD-L1 induces DNMT1-dependent DNA hypomethylation and restores the expression of methylationsilenced CDH1.

Inactivation of $N F_{\kappa} B$ blocks $P D-L 1 /$ Stat3/DNMT1 pathway in sorafenib-resistant HCC cells. To provide further insight into the mechanism underlying PD-L1/STAT3/DNMT1 signaling in sorafenib-resistant HCC, we considered the transcriptional factor $\mathrm{NF \kappa B}$ may play a key role. Indeed, as shown in Fig. 4A,

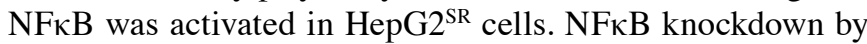
shRNA in HepG2 $2^{\text {SR }}$ cells significantly decreased PDL1 protein and mRNA levels followed by the dephosphorylation of STAT3 and downregulated DNMT1 expression (Fig. 4B). To elucidate 
A

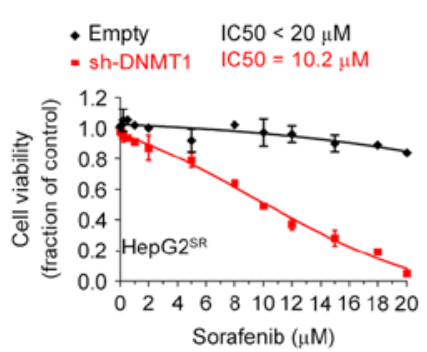

C

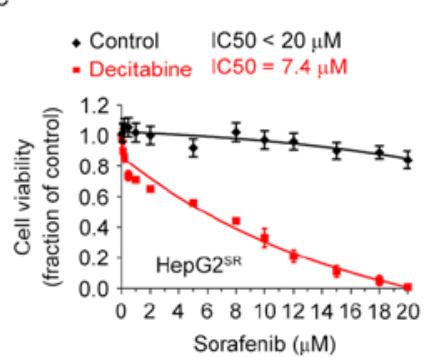



Sorafenib $(\mu \mathrm{M})$
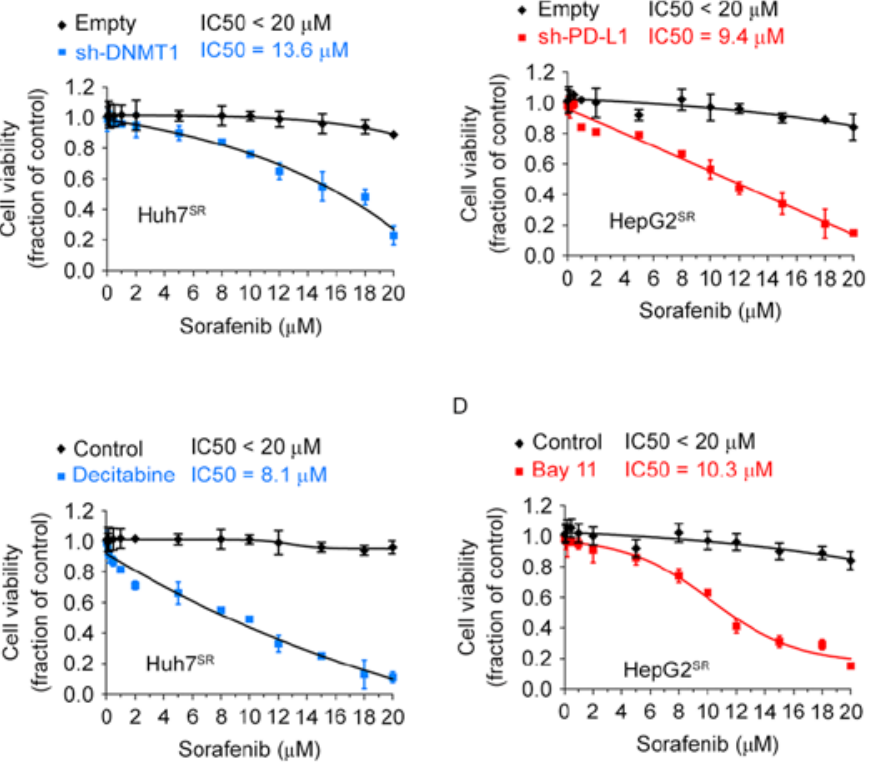

D

- Control IC50<20 $\mu \mathrm{M}$

- Bay $11 \quad$ IC $50=10.3 \mu \mathrm{M}$

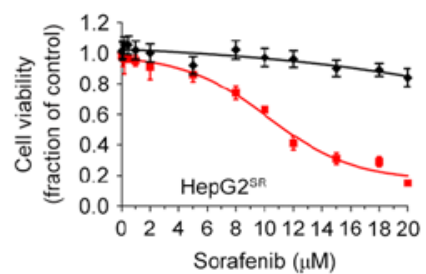

- Empty $\quad$ IC50 $<20 \mu \mathrm{M}$

- sh-PD-L1 IC50 = $9.9 \mu \mathrm{M}$

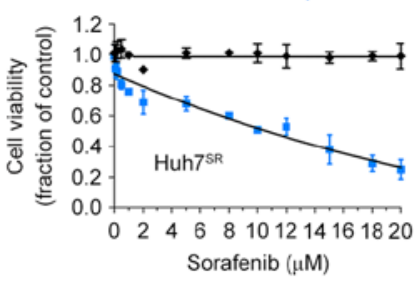

- Control IC50 $<20 \mu \mathrm{M}$ - Bay $11 \quad$ IC50 = 7.1 $\mu \mathrm{M}$

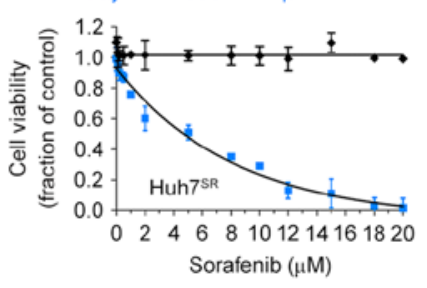

$E$
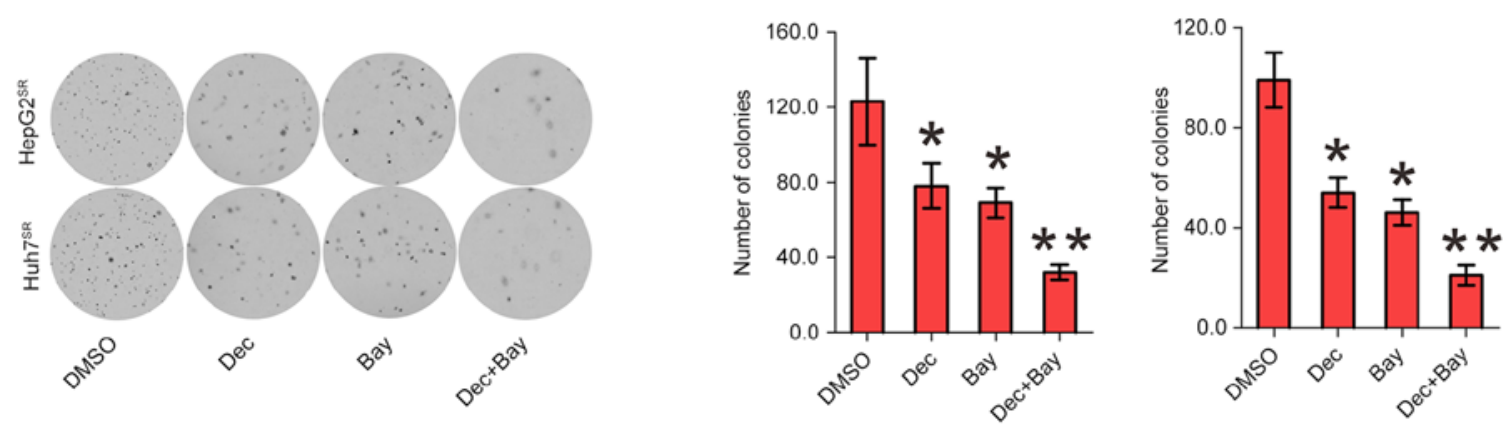

Figure 5. Genetic or pharmacological disruption of PD-L1 or DNMT1 sensitizes HCC resistance to sorafenib. (A) HepG2 ${ }^{\text {SR }}$ and Huh7 ${ }^{\text {SR }}$ cells were transfected with DNMT1 shRNA or empty vectors for $12 \mathrm{~h}$, treated with sorafenib for additional $72 \mathrm{~h}$ and subjected to CCK-8 assays. (B) HepG2 ${ }^{\text {SR }}$ and Huh7 ${ }^{\text {SR }}$ cells were transfected with PD-L1 shRNA or empty vectors for $12 \mathrm{~h}$, treated with sorafenib for additional $72 \mathrm{~h}$ and subjected to CCK-8 assays. (C) HepG2 ${ }^{\text {SR }}$ and Huh7 ${ }^{\text {SR }}$ cells were treated with decitabine for $12 \mathrm{~h}$, treated with sorafenib for additional $72 \mathrm{~h}$ and subjected to CCK-8 assays. (D) HepG2 ${ }^{\mathrm{SR}}$ and Huh $7^{\mathrm{SR}}$ cells were treated with Bay 11 for $12 \mathrm{~h}$, treated with sorafenib for additional $72 \mathrm{~h}$ and subjected to CCK-8 assays. (E) Resistant cells were treated with DMSO, decitabine, Bay 11 , or decitabine plus Bay 11 for $6 \mathrm{~h}$ then subjected to colony-forming assay. Representative images of colony-forming assay (left) and the quantification of colonies (right). Data are mean $\pm \mathrm{SD},{ }^{*} \mathrm{P}<0.05,{ }^{* *} \mathrm{P}<0.01$. The data represent three independent experiments.

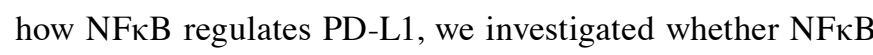
can directly transcriptionally regulate PDL1. Firstly, it was predicted that PDL1 promoter area has an NFאB binding site. Secondly, ChIP demonstrated $\sim 1$.5-fold of enrichment reduction of $\mathrm{NF} \kappa \mathrm{B}$ on PD-L1 promoter in $\mathrm{HepG} 2^{\mathrm{SR}}$ cells after $\mathrm{NF} \kappa \mathrm{B}$ shRNA transfection supporting that PD-L1 is one of the NFKB binding targets (Fig. 4C). Of note, as shown in Fig. 4D, when HepG2 $2^{\text {SR }}$ cells were treated at 1 and $3 \mathrm{M}$ of Bay 11, a specific NFאB inhibitor (22), the mRNA level of PD-L1 and DNMT1 were significantly reduced, and the $\mathrm{CDH} 1$ was markedly increased. These data suggest that inactivation of NFאB blocks the PD-L1/Stat3/DNMT1 pathway in sorafenib-resistance HCC cells.

Genetic or pharmacological disruption of PD-L1 or DNMT1 sensitizes HCC resistance to sorafenib. Because of activation of PD-L1 and DNMT1 signaling in sorafenib resistance to $\mathrm{HCC}$, we speculated that genetic or pharmacological disruption of PD-L1 and DNMT1 function could be an alternative strategy to impair sorafenib-resistant cell proliferation. When PD-L1 or DNMT1 was depleted by their specific shRNA in sorafenib-resistant cells, then exposing the cells to $0-20 \mu \mathrm{M}$ sorafenib for $72 \mathrm{~h}$, cell growth $\mathrm{IC}_{50}$ value for PD-L1 or DNMT1 knockdown was significantly decreased, respectively, compared with their empty control, suggesting that abrogation of PDL1 and DNMT1 restores sorafenib sensitivity (Fig. 5A and B). Next, we considered pharmacological inhibition using their inhibitor to sensitize resistant cells to sorafenib treatment. As shown in Fig. 5C, treatment with decitabine, a DNMT1 inhibitor, induced more pronounced inhibition on cell proliferation rate in HepG $2^{\text {SR }}$ and Huh7 ${ }^{\text {SR }}$. Because anti-PD-L1 can not inhibit PD-L1 expression but activity. Therefore, we consider Bay 11 can be used as PD-L1 inhibitor (Fig. 4D). Indeed, $3 \mathrm{M}$ of Bay 11 induced sensitized sorafenib-resistant cells (Fig. 5D). Decitabine and Bay 11 sensitized resistant cells to sorafenib treatment alone, and in a potential cooperation of NFאB/PDL1/DNMT1 in controlling sorafenib-resistant cell 
growth. We treated with decitabine and Bay 11 alone or both, and colony assays showed combination of decitabine and Bay 11 significantly disrupted the colony forming capability of HepG $2^{\text {SR }}$ and Huh7 ${ }^{\text {SR }}$ cells (Fig. 5E). Collectively, these data support the potential targeted combination therapies to enhance current and emerging sorafenib therapies for HCC.

\section{Discussion}

Hepatocellular cancer (HCC) is a leading cause of cancerrelated death worldwide, and the incidence of $\mathrm{HCC}$ is increasing in many parts of the world (23). Sorafenib is one of multiple targeted agents utilized by oncologists, and is FDA approved for treatment of a wide range of human cancers, including kidney, melanoma, prostate, ovarian, pancreatic, lung cancer and HCC. However, only rarely do sorafenib treated tumors regress completely, and the therapeutic effects of the drug are often temporary, most patients ultimately develop drug resistance and suffer relapses (24). Therefore, more effective therapeutic strategies and precision medicines to improve the prognosis of HCC patients with sorafenib resistance are urgently needed. Recent studies have shown that some mechanism was found in sorafenib-resistant cell lines. A tumor suppressor gene angiopoietin-like protein 1 (ANGPTL1) positively correlates with sorafenib sensitivity in HCC cells and human HCC tissues. ANGPTL1 directly interacts with and inactivates the MET receptor, which contributes to Slug suppression through inhibition of the extracellular receptor kinase/protein kinase B (ERK/AKT)-dependent early growth response protein 1 (Egr-1) pathway. ANGPTL1 may serve as a novel MET receptor inhibitor for advanced HCC therapy (25). Another study found PROX1 positively correlates with sorafenib resistance in HCC cells and may be a new potential strategy for improving sorafenib efficacy (26).

PD-L1, a ligand of PD1, is highly upregulated on many kinds of tumor cells, including melanoma, ovarian, and lung cancers. Because programmed death-1 (PD-1) and PD-L1 blockade have yielded promising clinical effects, understanding the regulatory mechanism of PD-L1 may identify biomarkers and/ or develop combinatorial strategies for clinical use. Recently, PD-L1 overexpression also was reported in resistant cells. PD-L1 was expressed at a high level in SCLC cells (H69 and H82) resistant to cisplatin versus parental cells (10). Bishop et al showed that ENZ resistance is associated with high frequency of PD-1/L1 therapy targets, both in the tumor and circulating immune cells (9). Cancer epigenetics involves the causes of $\mathrm{CpG}$ island hypermethylation in tumor suppressor genes leading to transcriptional silencing. DNMT1 is a member of DNA methyltransferase family which includes DNMT1, DNMT3a and DNMT3b. Methylation occurs predominantly at cytosine-C5 in the context of $\mathrm{CpG}$ dinucleotides, and is established and maintained by three DNA methyltransferases, DNMT3a, DNMT3b, and DNMT1. DNMT3a and DNMT3b have mostly de novo DNA methylation activity, whereas DNMT1 plays a central role in preserving the patterns of DNA methylation through cell division (27). DNMT1 and DNA methylation is often considered as a key epigenetic regulatory mechanism and potential target for resistance to drugs (28).

Although PD-L1 and DNMT1 has been shown to be overexpressed in human cancer and drug resistance, respectively, whether and how PD-L1/DNMT1 axis affect sorafenib resistance to $\mathrm{HCC}$ remains largely unexplored. In this study, we mainly focused on DNMT1, because GEO data analysis showed DNMT1 overexpression in sorafenib resistance HCC cells, but not DNMT3a and 3b (data not shown). In vitro study found DNMT1 was upregulated in sorafenibresistant HCC cells, which is consistent to GEO assay. Given both DNMT1 and PDL1 were significantly upregulated in sorafenib-resistant HCC cells and positively correlated by GEO assay, we proposed PD-L1 augments DNMT1 expression. We demonstrated that downregulation of PD-L1 in sorafenib-resistant cells might result from deregulation of DNMT1 at both protein and mRNA levels, leading to the upregulation of tumor suppressor $\mathrm{CDH} 1$ and the reduction of CDH1 promoter methylation. Moreover, depletion of PD-L1 or DNMT1 enhanced cell growth arrest, suggesting that PD-L1/DNMT1 axis plays a key role in survival and proliferation of sorafenib-resistant HCC cells. Accordingly, inhibition of PD-L1/DNMT1 axis may contribute to the resistance of molecular-targeted therapy. DNMT1 inhibitor caused a gradual decrease in self-renewal and tumorigenicity, and upregulation of apoptosis- and differentiation-related genes in HCC $(29,30)$. Decitabine is a useful demethylation agent in clinic. It can decrease DNMT1 expression and cell cycle and is commonly used as a single agent to treat patients with MDS and elderly patients with AML (30,31). PDL1 antibodies, such as durvalumab and avelumab, are selective, high-affinity, human antibodies that block PD-L1 binding to PD-1, allowing T cells to recognize and kill tumor cells $(7,32)$. However, these antibodies can only inhibit PD-L1 activity but not expression, which limits the use to overcome HCC resistance to sorafenib. Some transcranial factors were reported to directly regulate PD-L1. MYC regulates CD47 and PD-L1 at the transcriptional level by binding to their promoters in human melanoma (13). Moreover, PD-L1 is a direct target of HIF1- $\alpha$ and blockade of PD-L1 under hypoxia enhanced myeloid-derived suppressor cell-mediated $\mathrm{T}$ cell activation. Lo et al showed that inflammation increases PD-L1 expression in tumors through TNF- $\alpha$-mediated activation of $\mathrm{NF \kappa B}$, leading to transactivation of CSN5 (14). In this study, upregulation of phosphorylation of NFkB-p65 (Ser536) was positively associated with PD-L1 expression in sorafenib resistance HCC cells. Because NFKB knockdown significantly decreased both protein and mRNA levels of PD-L1 and DNMT1, especially, diminishing the binding of PD-L1 promoter, it demonstrated that PD-L1 is one of a direct target of $\mathrm{NF \kappa B}$ in sorafenib-resistant cells. Notably, $\mathrm{NF} \kappa \mathrm{B}$ inhibitor Bay-11 can decrease PD-L1 expression, which can be used to treat resistant cells to a PD-L1 inhibitor. Cell proliferation results showed that both decitabine and Bay 11 sensitize resistant cells to sorafenib treatment, further supporting that cellular function of PD-L1/DNMT1 signaling is required to sustain survival and proliferation of sorafenibresistant cells. Importantly, combination of decitabine and Bay 11 synergistically inhibited colony formation of HCC resistant cells to sorafenib indicating that dual inactivation of PD-L1 and DNMT1 synergistically disrupts the cell growth and proliferation of sorafenib-resistant HCC cells.

For mechanism study, first we elucidated how PD-L1 regulates the DNMT1 gene. As phosphorylated STAT3 can 
bind specific DNA elements resulting in transcriptional activation. Previously it was reported that STAT3 binds DNMT1 promoter and positively regulates DNMT1 transcription (33), we proposed that PD-L1 augments DNMT1 expression by STAT3 in sorafenib-resistant HCC cells. We found depletion of PD-L1 significantly downregulated phosphorylation of STAT3 followed by the DNMT1 expression. Then CHIP and promoter reporter assay confirmed that STAT3 transcriptionally regulate DNMT1. Of note, it is likely that STAT3 is downstream of PD-L1. One report suggests that STAT3 as an upstream target regulates PD-L1 expression (34). There may be a feedback loop between STAT3 and PD-L1 which need further investigation.

Taken together, our results identified a mechanistic and functional link between PD-L1 and DNMT1-dependent DNA methylation in HCC cells resistant to sorafenib. These results also established the clinical potential strategy for targeting $\mathrm{NF} \kappa \mathrm{B} / \mathrm{PD}-\mathrm{L} 1 / \mathrm{STAT} 3 / \mathrm{DNMT} 1$ axis aimed to improve sorafenib efficacy and overcoming sorafenib resistance.

\section{Acknowledgments}

The authors would like to thank Dr Ningling Kang at the Hormel Institute, University of Minnesota for providing HCC cell lines, and Lin Yang at the Mayo Clinic, Rochester, MN, USA for assistance in English editing. This study was supported by grants from Foundation of Jilin Provincial Development and Reform Commission (KY20160002; 3J115AJ73428, Yahui Liu), Jilin University Fund for Excellent Young Teacher (no. 419080500355, Bai Ji), and Youth Fund from the Department of Science and Technology, Jilin Province, China (no. 20140520026JH, Bai Ji).

\section{References}

1. Di Maio M, Daniele B and Perrone F: Targeted therapies: Role of sorafenib in HCC patients with compromised liver function. Nat Rev Clin Oncol 6: 505-506, 2009.

2. Wilhelm S, Carter C, Lynch M, Lowinger T, Dumas J, Smith RA, Schwartz B, Simantov R and Kelley S: Discovery and development of sorafenib: A multikinase inhibitor for treating cancer. Nat Rev Drug Discov 5: 835-844, 2006.

3. Sun X, Niu X, Chen R, He W, Chen D, Kang R and Tang D: Metallothionein-1G facilitates sorafenib resistance through inhibition of ferroptosis. Hepatology 64: 488-500, 2016.

4. Rudalska R, Dauch D, Longerich T, McJunkin K, Wuestefeld T, Kang TW, Hohmeyer A, Pesic M, Leibold J, von Thun A, et al: In vivo RNAi screening identifies a mechanism of sorafenib resistance in liver cancer. Nat Med 20: 1138-1146, 2014.

5. Zhou SL, Zhou ZJ, Hu ZQ, Huang XW, Wang Z, Chen EB, Fan J, Cao Y, Dai Z and Zhou J.. Tumor-associated neutrophils recruit macrophages and T-regulatory cells to promote progression of hepatocellular carcinoma and resistance to sorafenib. Gastroenterology 150: 1646-1658.e17, 2016.

6. Goodman A, Patel SP and Kurzrock R: PD-1-PD-L1 immunecheckpoint blockade in B-cell lymphomas. Nat Rev Clin Oncol 14: 203-220, 2017.

7. Massard C, Gordon MS, Sharma S, Rafii S, Wainberg ZA, Luke J, Curiel TJ, Colon-Otero G, Hamid O, Sanborn RE, et al: Safety and efficacy of durvalumab (MEDI4736), an anti-programmed cell death ligand-1 immune checkpoint inhibitor, in patients with advanced urothelial bladder cancer. J Clin Oncol 34: 3119-3125, 2016.

8. Reck M, Rodríguez-Abreu D, Robinson AG, Hui R, Csőszi T, Fülöp A, Gottfried M, Peled N, Tafreshi A, Cuffe S, et al; KEYNOTE-024 Investigators: Pembrolizumab versus chemotherapy for PD-L1-positive non-small-cell lung cancer. N Engl J Med 375: 1823-1833, 2016.
9. Bishop JL, Sio A, Angeles A, Roberts ME, Azad AA, Chi KN and Zoubeidi A: PD-L1 is highly expressed in Enzalutamideresistant prostate cancer. Oncotarget 6: 234-242, 2015.

10. Yan F, Pang J, Peng Y, Molina JR, Yang P and Liu S: Elevated cellular PD1/PD-L1 expression confers acquired resistance to cisplatin in small cell lung cancer cells. PLoS One 11: e0162925, 2016.

11. Zhu H, Bengsch F, Svoronos N, Rutkowski MR, Bitler BG, Allegrezza MJ, Yokoyama Y, Kossenkov AV, Bradner JE, Conejo-Garcia JR, et al: BET Bromodomain inhibition promotes anti-tumor immunity by suppressing PD-L1 expression. Cell Rep 16: 2829-2837, 2016.

12. Noman MZ, Desantis G, Janji B, Hasmim M, Karray S, Dessen P, Bronte V and Chouaib S: PD-L1 is a novel direct target of HIF-1 $\alpha$, and its blockade under hypoxia enhanced MDSC-mediated T cell activation. J Exp Med 211: 781-790, 2014.

13. Casey SC, Tong L, Li Y, Do R, Walz S, Fitzgerald KN, Gouw AM, Baylot V, Gütgemann I, Eilers M, et al: MYC regulates the antitumor immune response through CD47 and PD-L1. Science 352: 227-231, 2016.

14. Lo J, Lau EY, Ching RH, Cheng BY, Ma MK, Ng IO and Lee TK: Nuclear factor kappa B-mediated CD47 up-regulation promotes sorafenib resistance and its blockade synergizes the effect of sorafenib in hepatocellular carcinoma in mice. Hepatology 62: 534-545, 2015.

15. Baylin SB: DNA methylation and gene silencing in cancer. Nat Clin Pract Oncol 2 (Suppl 1): S4-S11, 2005.

16. Esteller M: Epigenetics in cancer. N Engl J Med 358: 1148-1159, 2008.

17. Esteller M, Fraga MF, Paz MF, Campo E, Colomer D, Novo FJ, Calasanz MJ, Galm O, Guo M, Benitez J, et al: Cancer epigenetics and methylation. Science 297: 1807-1808, 2002.

18. Spinzi G and Paggi S: Sorafenib in advanced hepatocellular carcinoma. N Engl J Med 359: 2497-2498, author reply 2498-2499, 2008.

19. Lee HJ, Zhuang G, Cao Y, Du P, Kim HJ and Settleman J: Drug resistance via feedback activation of Stat 3 in oncogene-addicted cancer cells. Cancer Cell 26: 207-221, 2014.

20. Zhang C, Li J, Huang T, Duan S, Dai D, Jiang D, Sui X, Li D, Chen Y, Ding F, et al: Meta-analysis of DNA methylation biomarkers in hepatocellular carcinoma. Oncotarget 7: 81255-81267, 2016.

21. Huang J, Wang Y, Guo Y and Sun S: Down-regulated microRNA-152 induces aberrant DNA methylation in hepatitis B virus-related hepatocellular carcinoma by targeting DNA methyltransferase 1. Hepatology 52: 60-70, 2010.

22. Chen L, Ruan Y, Wang X, Min L, Shen Z, Sun Y and Qin X: BAY 11-7082, a nuclear factor- $\kappa$ B inhibitor, induces apoptosis and S phase arrest in gastric cancer cells. J Gastroenterol 49: 864-874, 2014.

23. Llovet JM, Villanueva A, Lachenmayer A and Finn RS: Advances in targeted therapies for hepatocellular carcinoma in the genomic era. Nat Rev Clin Oncol 12: 436, 2015.

24. Chen J, Jin R, Zhao J, Liu J, Ying H, Yan H, Zhou S, Liang Y, Huang D, Liang X, et al: Potential molecular, cellular and microenvironmental mechanism of sorafenib resistance in hepatocellular carcinoma. Cancer Lett 367: 1-11, 2015.

25. Chen HA, Kuo TC, Tseng CF, Ma JT, Yang ST, Yen CJ, Yang CY, Sung SY and Su JL: Angiopoietin-like protein 1 antagonizes MET receptor activity to repress sorafenib resistance and cancer stemness in hepatocellular carcinoma. Hepatology 64: 1637-1651, 2016.

26. Liu Y, Ye X, Zhang JB, Ouyang H, Shen Z, Wu Y, Wang W, Wu J, Tao S, Yang X, et al: PROX1 promotes hepatocellular carcinoma proliferation and sorafenib resistance by enhancing $\beta$-catenin expression and nuclear translocation. Oncogene 34: 5524-5535, 2015.

27. Jin B and Robertson KD: DNA methyltransferases, DNA damage repair, and cancer. Adv Exp Med Biol 754: 3-29, 2013.

28. Min HY, Lee SC, Woo JK, Jung HJ, Park KH, Jeong HM, Hyun SY, Cho J, Lee W, Park JE, et al: Essential role of DNA methyltransferase 1-mediated transcription of insulin-like growth factor 2 in resistance to histone deacetylase inhibitors. Clin Cancer Res 23: 1299-1311, 2017.

29. Jiang $C$ and Gong F: DNA methyltransferase 1: A potential gene therapy target for hepatocellular carcinoma? Oncol Res Treat 39: 448-452, 2016.

30. Welch JS, Petti AA, Miller CA, Fronick CC, O'Laughlin M, Fulton RS, Wilson RK, Baty JD, Duncavage EJ, Tandon B, et al: TP53 and decitabine in acute myeloid leukemia and myelodysplastic syndromes. N Engl J Med 375: 2023-2036, 2016. 
31. Duncavage EJ, Uy GL, Petti AA, Miller CA, Lee YS, Tandon B, Gao F, Fronick CC, O'Laughlin M, Fulton RS, et al: Mutational landscape and response are conserved in peripheral blood of AML and MDS patients during decitabine therapy. Blood 129: 1397-1401, 2017.

32. Nagaya T, Nakamura Y, Sato K, Harada T, Choyke PL, Hodge JW, Schlom J and Kobayashi H: Near infrared photoimmunotherapy with avelumab, an anti-programmed death-ligand 1 (PD-L1) antibody. Oncotarget 8: 8807-8817, 2017.
33. Zhang Q, Wang HY, Woetmann A, Raghunath PN, Odum N and Wasik MA: STAT3 induces transcription of the DNA methyltransferase 1 gene (DNMT1) in malignant T lymphocytes. Blood 108: 1058-1064, 2006.

34. Wang WB, Yen ML, Liu KJ, Hsu PJ, Lin MH, Chen PM, Sudhir PR, Chen CH, Chen CH, Sytwu HK, et al: Interleukin-25 mediates transcriptional control of PD-L1 via STAT3 in multipotent human mesenchymal stromal cells (hMSCs) to suppress Th17 responses. Stem Cell Rep 5: 392-404, 2015. 